\title{
Panax Quinquefolium Saponins Attenuate Myocardial Dysfunction Induced by Chronic Ischemia
}

\author{
Ming Guo Jiangang Liu ${ }^{\mathrm{a}} \quad$ Feifei Guo ${ }^{\mathrm{b}} \quad$ Junhe Shic Chenglong Wang ${ }^{\mathrm{a}}$ \\ Paul W. Bible ${ }^{d}$ Minfu Yange Yi Tian ${ }^{f}$ Lai Weid $^{\mathrm{d}}$ Peili Wang $^{\mathrm{a}}$ \\ Dazhuo Shia \\ aCardiovascular Center, Xiyuan Hospital, China Academy of Chinese Medical Sciences, Beijing, \\ 'Institute of Chinese Materia Medica, China Academy of Chinese Medical Sciences, Beijing, China, \\ 'Department of Periodontics, University of Illinois at Chicago, Chicago, USA, 'State Key Laboratory \\ of Ophthalmology, Zhongshan Ophthalmic Center, Sun Yat-sen University, Guangzhou, eDepartment \\ of Nuclear Medicine, Beijing Chaoyang Hospital, Capital Medical University, Beijing, fDepartment of \\ Animal Experimental Center, State Key Laboratory of Cardiovascular Diseases, Fuwai Hospital, Chinese \\ Academy of Medical Sciences, Beijing, China
}

\section{Key Words}

Panax quinquefolium saponins $•$ Chronic myocardial ischemia $•$ Angiogenesis $•$ Proteomics

\begin{abstract}
Background/Aims: Previous studies in rat models of myocardial ischemia showed that Panax quinquefolium saponins (PQS) could attenuate ischemic/reperfusion injury, increase vessel density and improve cardiac function. In the current study, we examined whether PQS could attenuate myocardial dysfunction in a swine model of chronic myocardial ischemia (CMI). Methods: CMI was established in Bama mini-pigs by placing amroid constrictor on the left anterior descending artery (LAD). Starting from 2 months after the surgery, pigs randomly received $\mathrm{PQS}(30 \mathrm{mg} / \mathrm{kg} /$ day), atorvastatin $(1.5 \mathrm{mg} / \mathrm{kg} /$ day $)$, or no drug for one month $(\mathrm{n}=6)$. A group of pigs receiving sham surgery was included as an additional control. Glucose utilization was assessed with positron emission tomography-computer tomography (PET-CT). Cardiac function was assessed with echocardiography. Myocyte size, nuclear density, and arteriolar density were examined in tissue section obtained from the ischemia area. Potential molecular targets of PQS were identified using proteomic analysis with isobaric tags for relative and absolute quantitation (iTARQ) and network pharmacology. Results: In comparison to the sham controls, pigs implanted with ameroid constrictor had decreased ventricular wall motion, left ventricular ejection fraction (LVEF), and glucose utilization. PQS significantly increased cardiac function and glucose utilization. Arteriole density and myocyte nuclear density were increased. Myocyte diameter was decreased. PQS also attenuated the CMI-induced change of protein

M. Guo, F. Guo and L. Wei contributed equally to this work.

\begin{tabular}{ll}
\hline Peili Wang & Cardiovascular Center, Xiyuan Hospital, China Academy of Chinese Medical Sciences \\
and Dazhuo Shi & Beijing 100091 (China) \\
& Tel. +86 13911972856, Fax +86 62806155, E-Mail shidztcm@163.com; wangpeili_tcm@yeah.net
\end{tabular}
\end{abstract}




\section{Cellular Physiology Cell Physiol Biochem 2018;49:1277-1288 \begin{tabular}{l|l} 
DOI: 10.1159/000493407 & $\begin{array}{l}\text { @ } 2018 \text { The Author(s). Published by S. Karger AG, Basel } \\
\text { www.karger.com/cpb }\end{array}$ \\
\hline
\end{tabular}}

expression profile. The effects of atorvastatin were generally similar to that of PQS. However, PQS attenuated the reduction of left ventricular systolic WT induced by CMI more robustly than atorvastatin. Conclusion: The results from the current study supports the use of PQS in patients with coronary artery disease.

\section{Introduction}

Despite recent advancements in interventional therapies, including coronary artery bypass grafting (CABG) and percutaneous coronary interventions (PCI), coronary artery disease (CAD) remains the leading cause of cardiovascular morbidity and mortality worldwide. Approximately $30 \%$ of the patients with CAD suffer from recurrent attacks and are not eligible for CABG or PCI [1]. Inducing angiogenesis represents a promising therapeutic approach in such patients [2-4]. A variety of strategies, including growth factor, gene therapy, and cell-based therapies, have been shown to be effective in inducing angiogenesis in animal models of CAD, but translation into human use faces many challenges [5].

Statins, a group of selective inhibitors of 3-hydroxyl-3-methyl coenzyme A (HMG-CoA) reductase, are a widely used in patients with CAD to manage dyslipidemia. In addition to lipidlowering effects, statins also improve ventricular remodeling after myocardial infarction (MI), promote endothelial function, and reduce vascular inflammation [6-8]. A previous study using a swine model of CMI demonstrated that atorvastatin could promote angiogenesis [9]. In mouse models, atorvastatin could also promote angiogenesis and vascular maturation to enhance hematoma absorption [10]. Simvastatin, another member of the stain family, could promote angiogenesis via activation of Akt kinase and increasing nitrous oxide production in human umbilical vein endothelial cells (HUVECs) and endothelial progenitor cells $[11,12]$.

Panax quinquefolius saponins (PQS) are major active components of the stems and leaves of Panax quinquefolius (American ginseng), and has been approved for use (Z20030073) in human subjects for $>10$ years in China. A previous study from this laboratory demonstrated that PQS could increase the expression of VEGF and $\beta F G F$ in the ischemic area of myocardium in a rat model of acute myocardial infarction (AMI) [13]. As a step to move towards possible clinical use, we examined the effects of $\mathrm{PQS}$ on cardiac function and angiogenesis in a large animal model of CMI.

\section{Materials and Methods}

\section{Animals}

A total of 32 Bama pigs ( 4 months of age, $20 \pm 5 \mathrm{~kg}$; Tianjin Bainong Laboratory Animal Breeding Technology, SCXK [Tianjin] 2015-0002) were used in this study. Pigs were raised using standard chow and water in individual cages under strict veterinary supervision with normal circadian rhythm at $20 \pm 2{ }^{\circ} \mathrm{C}$. The study was approved by Animal Review Board of Chinese Academy of Medical Sciences [0082-1-40-G2(X)]. All experiments were conducted in compliance with the state legislations on the ethical use and care of laboratory animals.

\section{Surgical procedure}

Pigs were anaesthetized with ketamine hydrochloride $(20 \mathrm{mg} / \mathrm{kg}, \mathrm{im})$, propofol $(0.15 \mathrm{mg} / \mathrm{kg} / \mathrm{min}$, iv) and $0.5 \%-2 \%$ isoflurane-oxygen mixture (ventilation). The left anterior descending coronary artery (LAD) was exposed using left thoracotomy in sterile condition. Twenty-six pigs were implanted with ameroid constrictors; the remaining 6 pigs received sham surgery. Pigs received standard postoperative care that included the use of analgesics (fentanyl, $0.003 \mathrm{mg} / \mathrm{kg} \mathrm{q.d.).}$

Two months after the surgery, LAD stenosis was verified using coronary angiogram (Fig. S1a). LAD patency in the sham group was also verified (Fig. S1b). During the experiment, 4 pigs died during the surgery and another 4 died during anesthesia induction. The rate of accidental death (25\%) was consistent with that in previous studies [14].

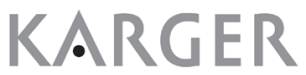




\section{Cellular Physiology Cell Physiol Biochem 2018;49:1277-1288 \begin{tabular}{l|l} 
and Biochemistry DOI: 10.1159/000493407 & $\begin{array}{l}\text { (c) 2018 The Author(s). Published by S. Karger AG, Basel } \\
\text { www.karger.com/cpb }\end{array}$
\end{tabular}

\section{Treatments}

At 2 months after the surgery, pigs implanted with ameroid constrictor were randomized to receive atorvastatin (1.5 mg/kg/day), PQS (30 mg/kg/day; from Yi-Sheng Pharmaceutical Co. Ltd., Jilin, China), or no drug for 1 month. Test drugs were mixed in pig chow. The amount of food intake was monitored daily to verify the dosage.

\section{Verification of major PQS constituents in peripheral blood}

Plasma concentration of major PQS constituents was determined with liquid chromatograph-mass spectrometry (LC-MS), as previously reported [15]. Representative results are shown in Fig. S2. The following five constituents were analyzed: pseudo-ginsenoside F11, ginsenoside Rc, ginsenoside Rb2, ginsenoside Rb3, ginsenoside Rd (Fig. S3).

\section{${ }^{18} \mathrm{~F}$-fluorodeoxyglucose-positron emission tomography (FDG-PET)}

At two month post-surgery (Baseline) and the end of drug treatment, FDG-PET was performed with a cardiac PET scanner (GE, Discovery, Boston, MA, USA). Briefly, pigs were anesthetized with ketamine and valium after overnight fasting. Six IU insulin was given intravenously to control the blood glucose. Twenty minutes later, ${ }^{18} \mathrm{~F}$-FDG ( $3 \mathrm{mCi}$ ) was administered intravenously. Scanning was carried out 1 hour later. The images were analyzed by QGS software (Cedars-Sinai Medical Center, Los Angeles, CA, USA). Standardized quantitative analysis was conducted with FDG-PET-bull's eye views, and mean signal intensity (MSI) was calculated using a 17-segment model [16] Manual fitting was conducted when left ventricular contour and mitral valve plane were impropriate for image interpretation. The results of analysis were obtained according to segmental scoring method standard solution (the American Heart Association) [17]. The max MSI segment was set to $100 \%$, and each segment was calculated automatically in accordance with the max MSI to reflect the regional glucose utilization. Glucose utilization was deemed to be low at MSI $<70 \%$. Summed rest score (SRS, the sum of the 17 segments), summed rest score percent (SRS\%), perfusion defect extent percent (PDE, \%), perfusion defect area (Defect, $\mathrm{cm}^{2}$ ) and total perfusion defect (TPD, \%) were acquired by QPS software. Images were assessed by two experienced experts independently.

\section{Echocardiography}

Two-dimensional echocardiography was performed to measure global and regional left ventricular function at the baseline and upon the completion of 1-month drug treatment using an ultrasound scanner (EPIQ7; Philips Medical Systems, Andover, MA). The global function of the left ventricular and the regional function of the myocardium were evaluated by left ventricular ejection fraction (LVEF) and systolic wall thickening (WT\%), respectively. LVEF was determined from the four-chamber views using a modified Simpson's algorithm. Regional wall thickness was evaluated at end systole and end diastole individually on two-dimensional echocardiograms. Left ventricular wall thickness at end-diastole (DWT) and end-systole (SWT) was measured to calculate the WT\% (SWT-DWT) / DWT $\times 100 \%$. The analysis was carried out by two experienced ultrasound technicians blinded to the treatment assignment independently.

\section{Tissue harvest and histological examination}

Pigs were euthanized with iv injection of saturated potassium chloride solution. The area at risk (AAR) in the LAD territory was collected. Remaining tissues were snap-frozen in liquid nitrogen.

\section{Arteriole density}

The AAR in the LAD territory was fixed in 4\% paraformaldehyde for $24 \mathrm{~h}$ and paraffin-embedded. Slices $(5 \mu \mathrm{m})$ were incubated with a mouse anti- $\alpha$ SMA (Lifespan, WA, USA) to identify arterioles. Anti-mouse IgG Fab2 Alexa Fluor (1:400) (Cell Signaling, Boston, MA) was used as secondary antibodies. Nuclei were counter-stained with DAPI. To determine arteriole density, 6 random fields per slide were captured at 200x magnification using a fluorescent microscope [18]. Blood vessels with a diameter $<100 \mu \mathrm{m}$ were counted by two independent examiners. Results are expressed as the number of vessels per field. 


\section{Cellular Physiology Cell Physiol Biochem 2018;49:1277-1288 \begin{tabular}{ll|l} 
DOI: 10.1159/000493407 & $\begin{array}{l}\text { @ } 2018 \text { The Author(s). Published by S. Karger AG, Basel } \\
\text { www.karger.com/cpb }\end{array}$ \\
\cline { 2 - 3 }
\end{tabular}

\section{Myocyte nuclear density and morphometry}

Periodic Acid-Schiff (PAS) stained sections were used to quantify myocyte diameter in AAR myocardium [19]. Myocyte diameter was calculated by counting at least 100 cells from AAR myocardium. Myocytes were counted regardless of size as long as myofilaments surrounding the nucleus could be identified. We also assessed myocyte nuclear density as previously described [20].

\section{Proteomic analysis}

Detailed proteomic operation and data analysis are described in supplementary material online (For all supplemental material see www.karger.com/10.1159/000493407/).

\section{Network pharmacology analysis}

Structural information of the 5 compounds in PQS were obtained from PubChem [21], and submitted to analysis using the BATMAN-TCM bioinformatic analysis platform [22]. This drug target prediction method ranks putative drug-target interactions based on their similarity to the known drug-target interactions [23]. Only the target proteins with top 1\% predictions score were considered (Table S1). Three validation strategies were applied to evaluate the performance of the prediction model [22]: "leave-one-interactionout" cross-validation, "leave-one-drug-out" cross-validation, and validation of the independent test set. Two types of nodes, including chemical compounds and the corresponding putative targets were predicted. Cytoscape (version 3.4.0) was utilized to visualize the drug/target interaction.

\section{Western blot}

Myocardium tissue in AAR (about $200 \mathrm{mg}$ ) was homogenized. Proteins were separated on 8\% SDSPAGE and transferred to PVDF membrane (Millipore, Temecula, CA, USA). The membrane was incubated overnight at $4^{\circ} \mathrm{C}$ with an antibody against PRKCD (1:1000, Lifespan Biotechnology) or GAPDH (1:1000, Santa Cruz Biotechnology, Dallas, TX, USA), and then with horseradish peroxidase-conjugated secondary antibody (1:1000) for 1 hour at room temperature. The images were captured using a Quantity One Image Analyzer (Bio-Rad, USA).

\section{Immunohistochemistry analysis}

Tissue sections were stained with an anti-PRKCD antibody (1:50, Lifespan Biotechnology), followed by an HRP-conjugated secondary antibody. Cells with brown granular diaminobenzidine (DAB) reaction in the cytoplasm were considered as positive.

\section{Statistical analysis}

Data are represented as mean \pm SEM and baseline data were analyzed using one-way analysis of variance (ANOVA), followed by Bonferroni posthoc analysis. Variables after drug treatment were analyzed with one-way analysis of covariance (ANCOVA). The SPSS (version 18.0; Chicago, IL) was used for analyses. $\mathrm{P}<0.05$ was considered statistically significant.

\section{Results}

PQS promoted cardiac function in pig mode of CMI

Echocardiography showed that, in comparison to the subjects receiving sham surgery, LVEF\% was significantly lowered in all subjects receiving the ameroid constrictor $(\mathrm{P}=0.001$ for both $\mathrm{PQS}$ and atorvastatin; $\mathrm{P}=0.0002$ for untreated; Fig. $1 \mathrm{a}$ ). In comparison to subjects receiving amroid constrictor but no drug treatment, both $\mathrm{PQS}$ and atorvastatin increased LVEF ( $\mathrm{P}=0.009$ and 0.018 for $\mathrm{PQS}$ and atorvastatin, respectively; Fig. 1b). Left ventricular systolic WT reduction induced by CMI was attenuated by both PQS and atorvastatin $(\mathrm{P}=0.00009$ and 0.045 for $\mathrm{PQS}$ and atorvastatin, respectively; Fig. 1c). The effect of $\mathrm{PQS}$ was more robust than atorvastatin $(\mathrm{P}=0.017)$. 
Fig. 1. Effects of $P Q S$ vs. atorvastatin on echocardiographic parameters. After two months, pigs subjected to sham operation or ameroid constrictor $\mathrm{u} \mathrm{n}$ d e r w e $\mathrm{n} \mathrm{t}$ echocardiography analysis for heart function. Pigs were subsequently treated with atorvastatin or PQS for one month. (a) Representative echocardiograms for determination of the LVEF\%. (bc) Quantitative measurement of LVEF and WT\%. Data are presented as mean \pm SEM; $* \mathrm{P}<0.05, \quad * * \mathrm{P}<0.01$; $\mathrm{n}=6$ per group.
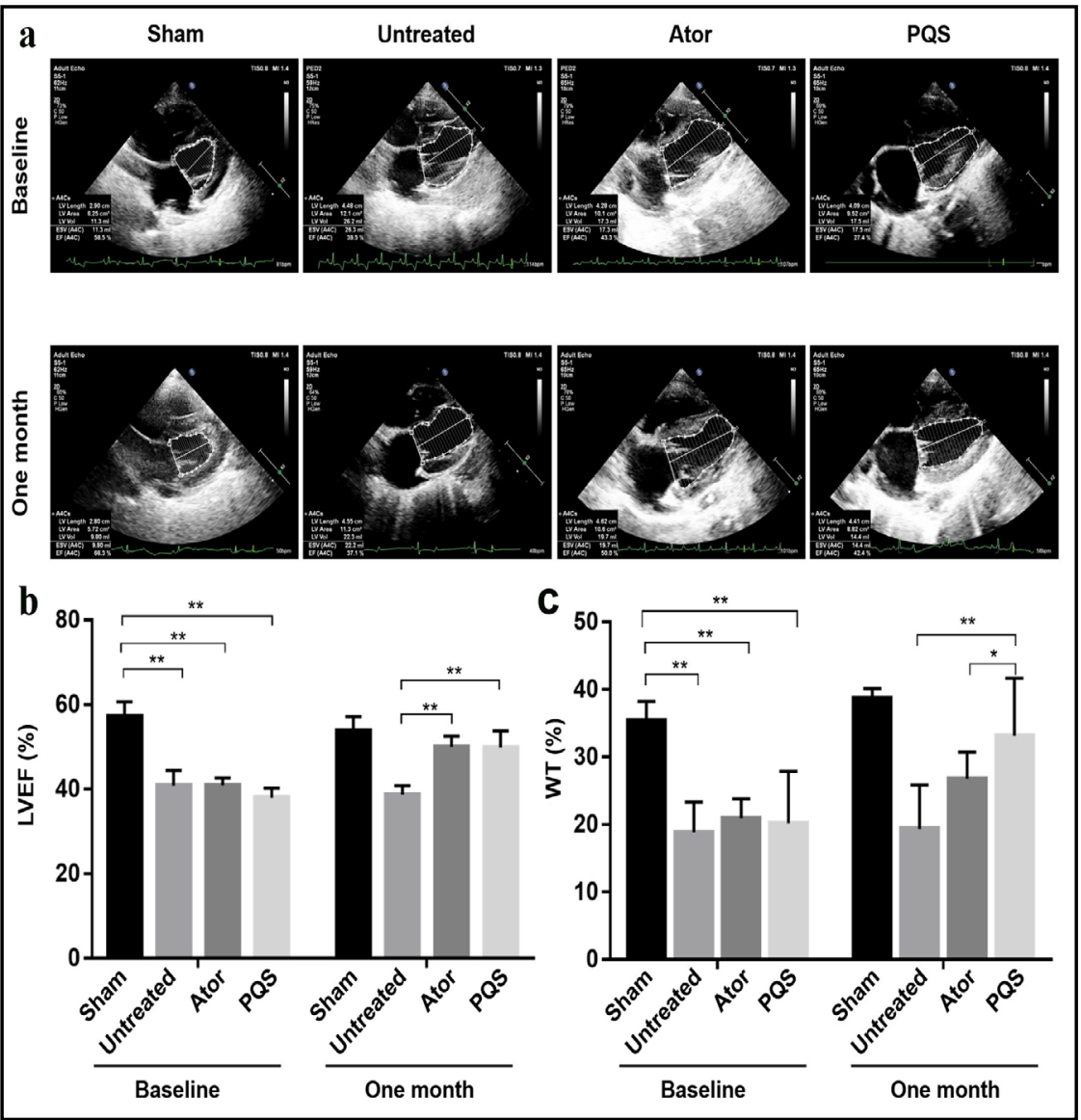

$P Q S$ improved myocardial glucose utilization in pig model of CMI

In comparison to subjects receiving sham surgery, SRS, SRS\%, $\mathrm{TPD} \%$ and $\mathrm{PDE} \%$ were significantly increased in subjects receiving ameroid constrictor after the surgery $(\mathrm{P}<0.0001$ for all, Fig. 2ad). SRS and TPD\% were decreased by PQS $(\mathrm{P}=0.012$ and 0.003 , respectively) as well as atorvastatin $(\mathrm{P}=0.047$ and 0.011, respectively). SRS\% and PDE\% were decreased by $P Q S(P=0.004$ and 0.013, respectively), but not by atorvastatin (Fig. $2 b / d)$.

CMI-induced reduction of MSI of mid-anteroseptal, apical septal, apical anterior, and apical inferior segments

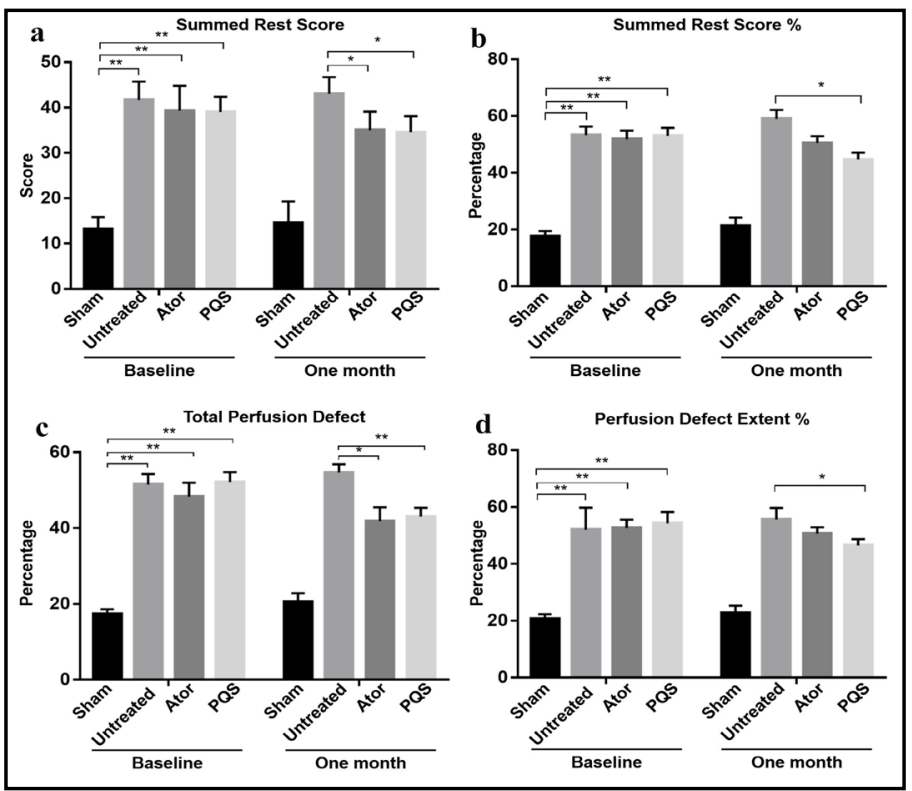

Fig. 2. Global ${ }^{18} \mathrm{~F}-\mathrm{FDG}$ PET imaging results(a-d) Quantitative measurement of SRS, SRS\%, TPD\% and perfusion defect extent $\%$. Data are presented as mean $\pm \mathrm{SEM} ;{ }^{*} \mathrm{P}<0.05,{ }^{* *} \mathrm{P}<0.01 ; \mathrm{n}=6$ per group. 


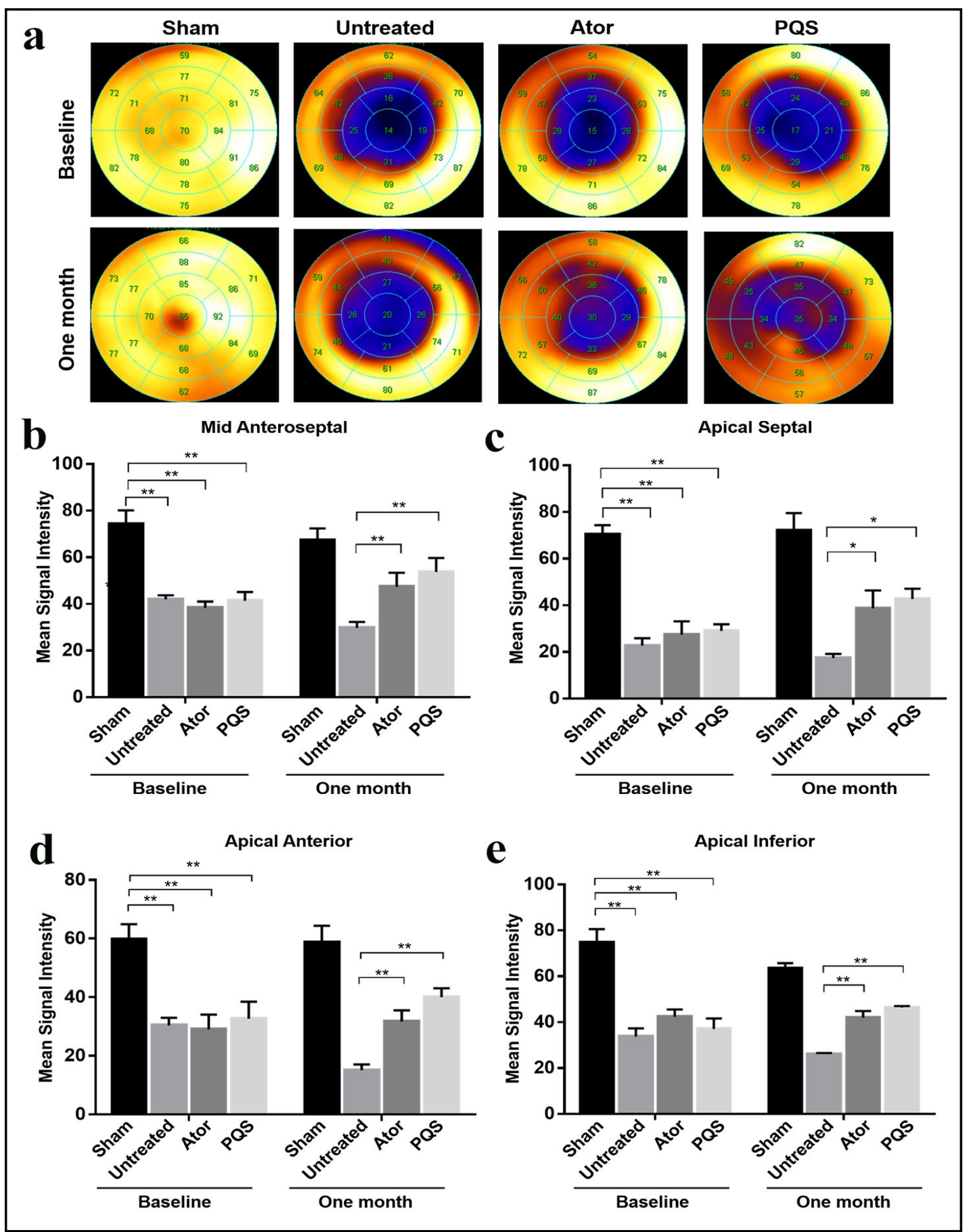

Fig. 3. Regional ${ }^{18} \mathrm{~F}-\mathrm{FDG}$ PET imaging results(a) Representative polar map of PET images. (b) Myocardial metabolism of left ventricular segmental MSI. Data are presented as mean $\pm \mathrm{SEM}$; ${ }^{*} \mathrm{P}<0.05 ;{ }^{* *} \mathrm{P}<0.01, \mathrm{n}=6$ per group. 
was attenuated by $\mathrm{PQS}$ $(\mathrm{P}=0.001, \quad 0.001, \quad \mathrm{P}=0.03$ and 0.000172 , respectively) as well as atorvastatin $(\mathrm{P}=0.004,0.005,0.045$ and 0.002 , respectively) (Fig. 3a-e).

$P Q S$ increased arteriole density and myocyte nuclear density, and reduced myocyte diameter

Arteriole density in the ischemic myocardium was increased by both $P Q S$ and atorvastatin $\quad(\mathrm{P}=0.00013$ and 0.009 , respectively; Fig. 4a). The arteriole number was significantly higher in subjects receiving $\mathrm{PQS}$ than with atorvastatin $(\mathrm{P}=0.045$, Fig. 4a/b).

PAS staining showed reduced myocyte nuclear density, with compensatory myocyte cellular hypertrophy, in ischemic myocardium after the implantation of ameroid constrictor (Fig. 5a). Both PQS and aotrvastatin increased myocyte nuclear density $(\mathrm{P}=0.001$ and 0.011 , respectively; Fig. 5a-b), with a statistically greater effect with $P Q S(P=0.048$ vs. atorvastatin; Fig. 5b). Myocyte diameter was reduced by $\mathrm{PQS}(\mathrm{P}=0.014)$, but not by atorvastatin $(\mathrm{P}=0.064$, Fig. 5c).

$P Q S$ reversed global protein expression changes caused by CMI

A total of 3, 470 proteins was quantified with mass spectrometry, among which 733 were differentially expressed between subjects with vs. without CMI: 341 proteins were upregulated and 392 proteins were downregulated by at least $20 \%(\mathrm{P}<0.05$, Student's t-test $)$

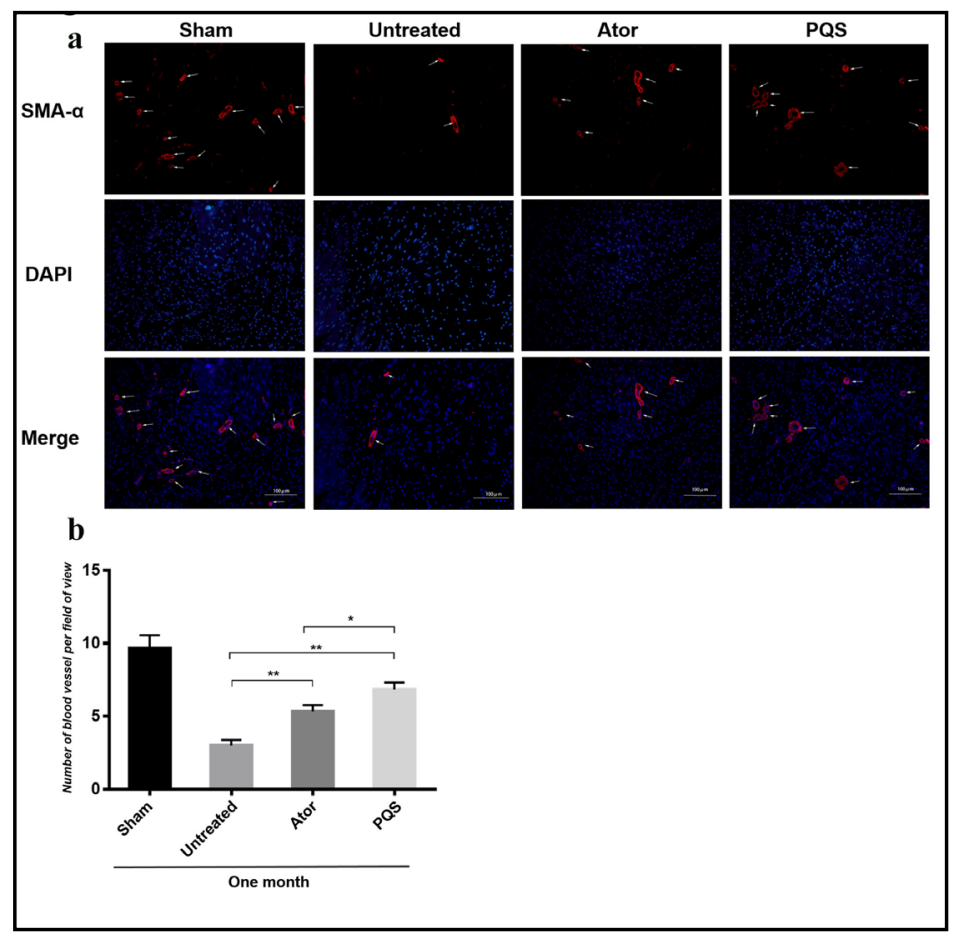

Fig. 4. Immunohistochemical analysis of arterioles in ischemic myocardium. (a) Representative images of $\alpha$-smooth muscle actin (SMA)-positive arterioles (red signals; scale bars: $100 \mu \mathrm{m}$ ). (b) Myocardial arterioles were quantified from 6 random fields $(\times 20$ magnification). Data are presented as mean $\pm \mathrm{SEM} ;{ }^{*} \mathrm{P}<0.05 ;{ }^{*} \mathrm{P}<0.01$, $\mathrm{n}=6$ per group.

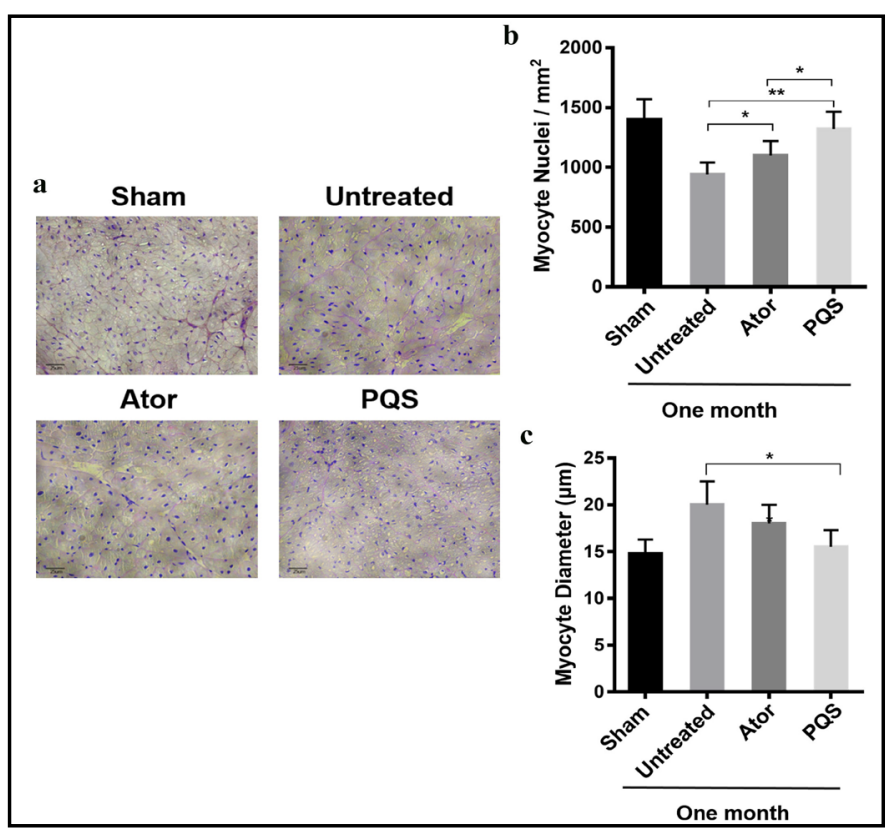

Fig. 5. Effects of PQS vs. atorvastatin on myocyte diameter and nuclear density. (a) PAS-stained sections from AAR of LAD territory. (b) Both PQS and atorvastatin increased myocyte nuclear density. (c) Reduction in myocyte diameter by PQS. Data are presented as mean $\pm \mathrm{SEM}$; ${ }^{*} \mathrm{P}<0.05 ;{ }^{* *} \mathrm{P}<0.01$ 
Fig. 6. Proteomic analysis of $P Q S$ effect on myocardium. (a) Venn diagram describing the numbers of proteins upregulated when comparing their expression in the untreated myocardium to sham myocardium and downregulated when comparing their expression in PQS treated myocardium to untreated myocardium. (b) Hierarchical clustering of 733 proteins with expression changes between untreated and sham myocardium. (c) Network pharmacology analysis showed five putative targets of the five major components of PQS. Green nodes represent 5 chemical components of PQS; blue nodes or pink node represent proteins which interact with chemical components and were differentially expressed in $\mathrm{PQS}$-treated sample; pink node also represents protein related with angiogenesis.

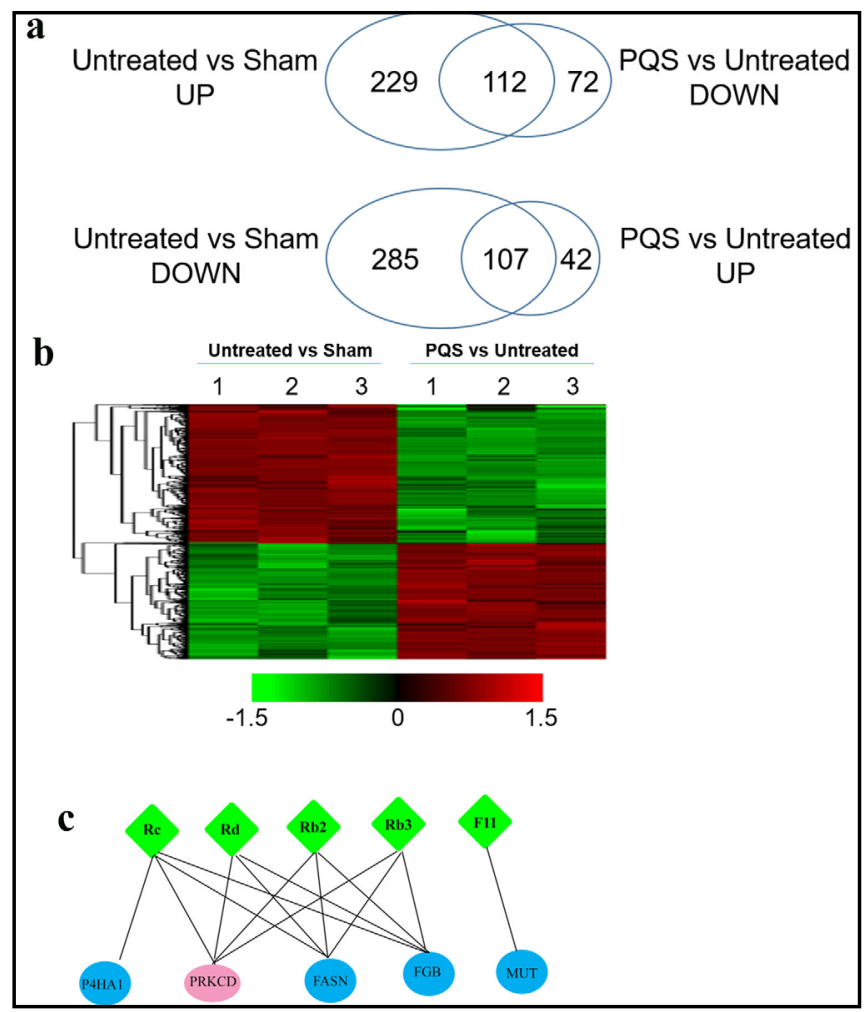

in ischemic myocardium (Fig. 6a). PQS treatment reversed the majority of these changes (Fig. 6b): only 5 out of the 341 upregulated proteins and 2 out of the 392 downregulated proteins remained statistically different than in subjects receiving sham surgery after 1-month PQS treatment (Table S2-S5).

Proteins that were differentially expressed between subjects with CMI vs. no CMI but restored by $\mathrm{PQS}$ treatment (n=219, Fig. 6a) were fed into a network pharmacology analysis using the similarity-based target prediction method BATMAN-TCM [22]. The analysis identified 5 putative protein targets of the 5 major PQS components (Rb2, Rb3, Rc, Rd, and F11), including PRKCD, P4HA1, FASN, FGB, and MUT (Fig. 6c).

PRKCD is the only protein among the 5 candidate targets of $\mathrm{PQS}$ that is known to block angiogenesis [24]. Western blot analysis showed increased expression of PRKCD in ischemic myocardium (vs. sham surgery; Fig. 7a); such an effect was reversed by PQS (Fig. 7b). Immunohistochemistry yielded identical results (Fig. 7c/d).

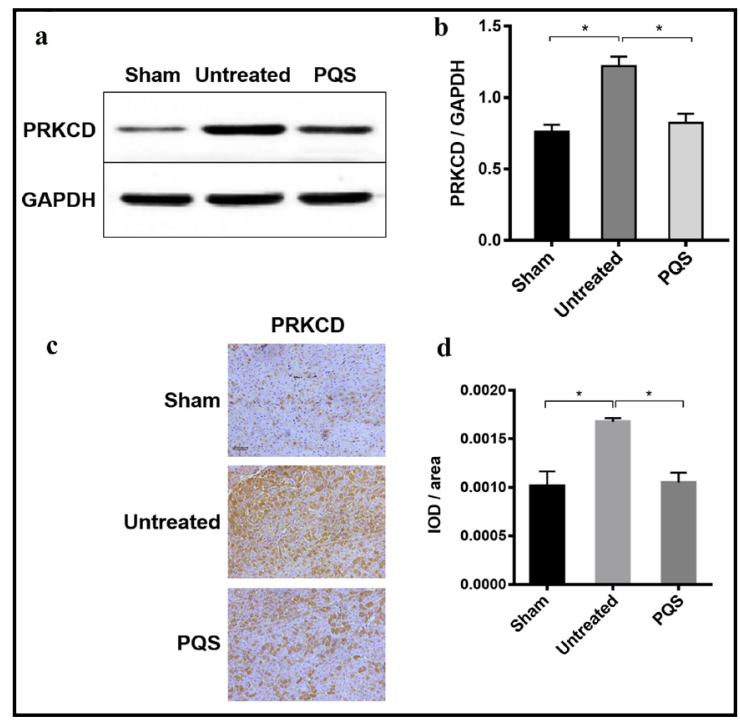

Fig. 7. PRKCD analysis with Western blot and immunohistochemical staining. (a) Representative Western immunoblots showing the effects of different treatment on PRKCD. (b) Graphical representation of the relative fold changes of proteins. (c) Representative immunohistochemical staining for PRKCD in the AAR myocardium (scale bar: $100 \mu \mathrm{m}$ ) (d) Quantification of the intensity of immunohistochemical staining. Data are presented as mean $\pm \mathrm{SEM} ;{ }^{*} \mathrm{P}<0.05 ;{ }^{*} \mathrm{P}<0.01, \mathrm{n}=6$ per group. 


\section{Cellular Physiology Cell Physiol Biochem 2018;49:1277-1288 \begin{tabular}{l|l} 
and Biochemistry Published online: 12 September, 2018 & $\begin{array}{l}\text { (C) } 2018 \text { The Author(s). Published by S. Karger AG, Basel } \\
\text { www.karger.com/cpb }\end{array}$
\end{tabular} \\ Guo et al.: Panax Quinquefolium Saponins for Chronic Myocardial Ischemia}

\section{Discussion}

To the best of our knowledge, this study represents the first study that demonstrated beneficial effects of PQS in a large animal model of CMI. The most important finding is improved myocardial function as reflected by increased LVEF\% and WT\% by both PQS and atorvastatin. The second major finding is improvement of glucose utilization in ischemic myocardium by both $\mathrm{PQS}$ and atorvastatin. Both $\mathrm{PQS}$ and atorvastatin increased density of arterioles and myocyte nuclei. However, cardiac hypertrophy, as reflected by myocyte diameter change, was ameliorated only by $\mathrm{PQS}$. Another preliminary but potentially important finding is the likely involvement of PRKCD in the reversal of CMI-induced protein expression changes by PQS.

${ }^{18}$ F-FDG PET imaging reflects the rate of cellular glucose uptake. Myocardial uptake of glucose is dependent on serum insulin [25]. Consistent with previous studies that showed decreased insulin-dependent glucose uptake by the heart [26], we showed decreased glucose uptake in mini pigs receiving ameroid constrictior. PQS clearly improved glucose utilization in ischemic area, as evidenced by decreased SRS, SRS\%, TPD\% and PDE\% in comparison to subjects with CMI but receiving no drug treatment. In addition, MSI in all four measured segments (e.g.,mid-anteroseptal, apical septal, apical anterior, and apical inferior) was increased significantly by PQS, indicating improved regional glucose metabolism.

Neovascularization and remodeling of the preexisting vasculature are hallmark features in CMI. Remodeling is regulated by local pro-angiogenic factors, inflammatory reactions and vascular progenitor cells, and critically affects the long-term functional outcomes of the ischemic area [27]. Promoting myocardial angiogenesis is a promising approach for the treatment of CMI [28]. In the current study, arteriole density was significantly increased by both $\mathrm{PQS}$ and atorvastatin, thus supporting the functional improvement of myocardial function. It is noteworthy that the magnitude of change in arteriole density was greater with PQS treatment than with atorvastatin. This may explain the greater improvement in WT\% and SRS\% with PQS. Consistent with previous studies [18, 29], myocyte nuclear density was increased by PQS. Treatment with PQS attenuated the cellular hypertrophy, indicating that PQS improve oxygen transport across the sarcolemma in the ischemic myocardium [30].

$\mathrm{PQS}$ treatment altered the global protein expression in AAR myocardium. Such effects were generally minor (between -2.2 and 2 fold). Majority of the changes induced by CMI was reversed by $\mathrm{PQS}$, suggesting that $\mathrm{PQS}$ exerts its action via multiple targets that individually may not be sufficient to produce any meaningful benefits.

PKC, a member of a large family of serine/threonine-specific protein kinase, is associated with pathophysiology of vascular complication [31] and can be activated by signals such as overloading $\mathrm{Ca}^{2+}$ or diacylglycerol. PRKCD, known as PKC $\delta$ in porcine species, belongs to the PKC family and is expressed in endothelial cells (ECs) and smooth muscle cells (SMCs) $[32,33]$. A study in rats demonstrated that PKC $\delta$ could impair vessel formation in diabetic ischemic limbs [24], suggesting that PKC $\delta$ is a negative regulator of angiogenesis. In our study, we showed PQS could reverse CMI-induced elevation in PRKCD expression, indicating that PQS may promote angiogenesis through blocking the expression of PRKCD.

The current study has several limitations. First, recent studies suggest that heart is capable of self-regeneration [34]. This, continuous growth of the mini-pigs used in the current study could be a confounding factor that contributed to the improvement of cardiac function and myocardial metabolism. Second, the treatment period was only 1 month in the current study. Long-term effect of $\mathrm{PQS}$ on cardiac function needed to be investigated in the future. Third, previous studies had demonstrated PQS could interact with phenelzine and warfarin [35]. The current study did not examine whether PQS and atorvastatin interact at a pharmacokinetic level. As a result, further studies are needed before PQS could be used in subjects also receiving atorvastatin Lastly, analysis with BATMAN-TCM only provided a preliminary set of potential targets. These targets require validation and further investigation. 


\section{Cellular Physiology Cell Physiol Biochem 2018;49:1277-1288 \begin{tabular}{ll|l} 
DOI: 10.1159/000493407 & $\begin{array}{l}\text { O } 2018 \text { The Author(s). Published by S. Karger AG, Basel } \\
\text { www.karger.com/cpb }\end{array}$ \\
\hline
\end{tabular}}

\section{Conclusion}

One month of $\mathrm{PQS}$ treatment could improve cardiac function and glucose utilization in a swine model of CMI, possibly by reversing global protein expression changes caused by CMI.

\section{Abbreviations}

AAR (area at risk); CAD (coronary artery disease); CMI (chronic myocardial ischemia); FDG-PET-CT (fluorodeoxy glucose-positron emission tomography-computer tomography); HMG-CoA (hydroxyl-methyl-glutaryl coenzyme A); HUVEC (human umbilical vein endothelial cells); iTARQ (isobaric tags for relative and absolute quantitation); LAD (left anterior descending artery); LC-MS (liquid chromatograph-mass spectrometry); LVEF (left ventricular ejection fraction); MI (myocardial infarction); MSI (mean signal intensity); PAS (periodic Acid-Schiff); PDE (perfusion defect extent); PQS (Panax quinquefolium saponins); SRS (summed rest score); TPD (total perfusion defect); WT (wall thickening).

\section{Acknowledgements}

We thank Baojie Zhang, Bo Ju, Haiying Song for technical help with animal model experiment and Junying Wei and Weiran Lin for performing the proteomics analysis.

This study was supported by the National Natural Sciences Foundation of China Grant (81473528), the Jilin Province Major Science and Technology Achievements Transformation Project Grant (11ZDZH005), the Foundation for Fostering Doctoral Innovative Talent (CX201709), and the National Basic Research Program of China (973 program, 2015CB554402).

\section{Disclosure Statement}

The authors have no conflict of interests to disclose.

\section{References}

1 Lassaletta AD, Chu LM, Sellke FW: Therapeutic neovascularization for coronary disease: current state and future prospects. Basic Res Cardiol 2011;106:897-909.

-2 Fujita M, Miyamoto S: Progress in the Functional Assessment of Human Coronary Collateral Circulation. Circ J 2012;76:297-298.

-3 Traupe T, Gloekler S, de Marchi SF, Werner GS, Seiler C: Assessment of the Human Coronary Collateral Circulation. Circulation 2010;122:1210-1220.

-4 Meier P, Lansky AJ, Fahy M, Xu K, White HD, Bertrand ME, Mehran R, Stone GW: The impact of the coronary collateral circulation on outcomes in patients with acute coronary syndromes: results from the ACUITY trial. Heart 2014;100:647-651.

-5 Mitsos S, Katsanos K, Koletsis E, Kagadis GC, Anastasiou N, Diamantopoulos A, Karnabatidis D, Dougenis D: Therapeutic angiogenesis for myocardial ischemia revisited: basic biological concepts and focus on latest clinical trials. Angiogenesis 2012;15:1-22.

6 Dupuis J, Tardif JC, Cernacek P, Theroux P: Cholesterol reduction rapidly improves endothelial function after acute coronary syndromes - The RECIFE (reduction of cholesterol in ischemia and function of the endothelium) trial. Circulation 1999;99:3227-3233.

7 Lefer AM, Campbell B, Shin YK, Scalia R, Hayward R, Lefer DJ: Simvastatin preserves the ischemicreperfused myocardium in normocholesterolemic rat hearts. Circulation 1999;100:178-184. 


\section{Cellular Physiology Cell Physiol Biochem 2018;49:1277-1288

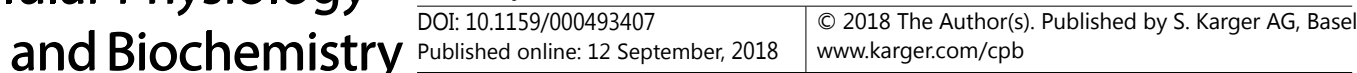

8 Pruefer D, Scalia R, Lefer AM: Simvastatin inhibits leukocyte-endothelial cell interactions and protects against inflammatory processes in normocholesterolemic rats. Arterioscler Thromb Vasc Biol 1999;19:2894-2900.

-9 Elmadhun NY, Lassaletta AD, Chu LM, Liu YH, Feng J, Sellke FW: Atorvastatin increases oxidative stress and modulates angiogenesis in Ossabaw swine with the metabolic syndrome. J Thorac Cardiovasc Surg 2012;144:1486-1493.

10 Wang D, Li T, Wei HJ, Wang Y, Yang GL, Tian Y, Zhao ZL, Wang L, Yu SP, Zhang YQ, Chen JL, Jiang RC, Zhang JN: Atorvastatin enhances angiogenesis to reduce subdural hematoma in a rat model. J Neurol Sci 2016;362:91-99.

-11 Kureishi Y, Luo ZY, Shiojima I, Bialik A, Fulton D, Lefer DJ, Sessa WC, Walsh K: The HMG-CoA reductase inhibitor simvastatin activates the protein kinase Akt and promotes angiogenesis in normocholesterolemic animals. Nat Med 2000;6:1004-1010.

12 Dimmeler S, Aicher A, Vasa M, Mildner-Rihm C, Adler K, Tiemann M, Rutten H, Fichtlscherer S, Martin H, Zeiher AM: HMG-CoA reductase inhibitors (statins) increase endothelial progenitor cells via the PI 3-kinase/Akt pathway. J Clin Invest 2001;108:391-397.

13 Wang C, Shi D, Yin H: Effect of panax quinquefolius saponin on angiogenesis and expressions of VEGF and bFGF in myocardium of rats with acute myocardial infarction. Chin J Integr Med 2007;27:331-334.

14 Canty JM, Suzuki G, Banas MD, Verheyen F, Borgers M, Fallavollita JA: Hibernating myocardium - Chronically adapted to ischemia but vulnerable to sudden death. Circ Res 2004;94:1142-1149.

15 Yang L, Miao Y, Yin H, Shi D, Chen K: Identification of ginsenosides from Panax quinquefolius in Xinyue Capsula by LC-ESI-MSn. Zhong cao yao 2010;41:1942-1947.

16 Cerqueira MD, Weissman NJ, Dilsizian V, Jacobs AK, Kaul S, Laskey WK, Pennell DJ, Rumberger JA, Ryan T, Verani MS, Amer Heart Assoc Writing G: Standardized myocardial segmentation and nomenclature for tomographic imaging of the heart - A statement for healthcare professionals from the Cardiac Imaging Committee of the Council on Clinical Cardiology of the American Heart Association. Circulation 2002;105:539-542.

17 Dobert N, Britten M, Assmus B, Berner U, Menzel C, Lehmann R, Hamscho N, Schachinger V, Dimmeler S, Zeiher AM, Grunwald F: Transplantation of progenitor cells after reperfused acute myocardial infarction: evaluation of perfusion and myocardial viability with FDG-PET and thalliurn SPECT. Eur J Nucl Med Mol Imaging 2004;31:1146-1151.

18 Giordano C, Thorn SL, Renaud JM, Al-Atassi T, Boodhwani M, Klein R, Kuraitis D, Dwivedi G, Zhang P, DaSilva JN: Pre-clinical evaluation of biopolymer-delivered circulating angiogenic cells in a swine model of hibernating myocardium. Circ Cardiovasc Imaging 2013;6:982-991.

19 Suzuki G, Lee TC, Fallavollita JA, Canty JM: Adenoviral gene transfer of FGF-5 to hibernating myocardium improves function and stimulates myocytes to hypertrophy and reenter the cell cycle. Circ Res 2005;96:767-775.

20 Lim HJ, Fallavollita JA, Hard R, Kerr CW, Canty JM: Profound apoptosis-mediated regional myocyte loss and compensatory hypertrophy in pigs with hibernating myocardium. Circulation 1999;100:2380-2386.

-21 Wang YL, Bryant SH, Cheng TJ, Wang JY, Gindulyte A, Shoemaker BA, Thiessen PA, He SQ, Zhang J: PubChem BioAssay: 2017 update. Nucleic Acids Res 2017;45:D955-D963.

-22 Liu ZY, Guo FF, Wang Y, Li C, Zhang XL, Li HL, Diao LH, Gu JY, Wang W, Li D, He FC: BATMAN-TCM: a Bioinformatics Analysis Tool for Molecular mechANism of Traditional Chinese Medicine. Sci Rep 2016;6:21146.

-23 Perlman L, Gottlieb A, Atias N, Ruppin E, Sharan R: Combining Drug and Gene Similarity Measures for DrugTarget Elucidation. J Comput Biol 2011;18:133-145.

-24 Lizotte F, Pare M, Denhez B, Leitges M, Guay A, Geraldes P: PKC delta Impaired Vessel Formation and Angiogenic Factor Expression in Diabetic Ischemic Limbs. Diabetes 2013;62:2948-2957.

25 Maki M, Luotolahti M, Nuutila P, Iida H, VoipioPulkki LM, Ruotsalainen U, Haaparanta M, Solin O, Hartiala J, Harkonen R, Knuuti J: Glucose uptake in the chronically dysfunctional but viable myocardium. Circulation 1996;93:1658-1666.

-26 Dilsizian V, Bacharach SL, Beanlands RS, Bergmann SR, Delbeke D, Dorbala S, Gropler RJ, Knuuti J, Schelbert HR, Travin MI: ASNC imaging guidelines/SNMMI procedure standard for positron emission tomography (PET) nuclear cardiology procedures. J Nucl Cardiol 2016;23:1187-1226. 


\section{Cellular Physiology Cell Physiol Biochem 2018;49:1277-1288 \begin{tabular}{ll|l} 
and Biochemistry $10.1159 / 000493407$ & Published online: 12 September, 2018 & $\begin{array}{l}\text { O } 2018 \text { The Author(s). Published by S. Karger AG, Basel } \\
\text { www.karger.com/cpb }\end{array}$ \\
\hline
\end{tabular}

27 Silvestre JS, Smadja DM, Levy BI: Postischemic revascularization: from cellular and molecular mechanisms to clinical applications. Physiol Rev 2013;93:1743-1802.

28 Deveza L, Choi J, Yang F: Therapeutic Angiogenesis for Treating Cardiovascular Diseases. Theranostics 2012;2:801-814.

29 Page BJ, Banas MD, Suzuki G, Weil BR, Young RF, Fallavollita JA, Palka BA, Canty JM: Revascularization of Chronic Hibernating Myocardium Stimulates Myocyte Proliferation and Partially Reverses Chronic Adaptations to Ischemia. J Am Coll Cardiol 2015;65:684-697.

-30 Dorn GW: Apoptotic and non-apoptotic programmed cardiomyocyte death in ventricular remodelling. Cardiovasc Res 2009;81:465-473.

-31 Dempsey EC, Newton AC, Mochly-Rosen D, Fields AP, Reyland ME, Insel PA, Messing RO: Protein kinase $\mathrm{C}$ isozymes and the regulation of diverse cell responses. Am J Physiol Lung Cell Mol Physiol 2000;279:L429-L438.

32 Shimizu K, Watanabe K, Yamashita H, Abe M, Yoshimatsu H, Ohta H, Sonoda H, Sato Y: Gene regulation of a novel angiogenesis inhibitor, vasohibin, in endothelial cells. Biochem Biophys Res Commun 2005;327:700706.

-33 Leitges M, Mayr M, Braun U, Mayr U, Li CH, Pfister G, Ghaffari-Tabrizi N, Baier G, Hu YH, Xu QB: Exacerbated vein graft arteriosclerosis in protein kinase C delta-null mice. J Clin Invest 2001;108:1505-1512.

34 Leri A, Kajstura J, Anversa P: Role of Cardiac Stem Cells in Cardiac Pathophysiology: A Paradigm Shift in Human Myocardial Biology. Circ Res 2011;109:941-961.

-35 Izzo AA, Ernst E: Interactions Between Herbal Medicines and Prescribed Drugs An Updated Systematic Review. Drugs 2009;69:1777-1798. 